\title{
Micronutrients against oxidative stress - time for clinical recommendations?
}

\author{
Jan Wernerman* \\ See related research by Manzanares et al., http://ccforum.com/content/16/2/R66/abstract
}

\begin{abstract}
Antioxidant micronutrient supplementation may be beneficial for critically ill patients. Often, cocktails of antioxidant micronutrients are used. Consequently, meta-analyses of available randomized controlled trials of antioxidant micronutrient supplementation are of particular interest. Because the majority of randomized controlled trials included in these meta-analyses use a combination of several antioxidant micronutrients, conclusions are difficult to draw. The scientific step to take now is to gain more knowledge about antioxidant mechanisms by coupling plasma concentrations to effects and outcomes.
\end{abstract}

Oxidative stress is the imbalance between oxidants and antioxidants leading to damage' [1]. This is suggested as pathogenesis in a wide range of conditions. The toxicity of a high concentration of oxygen in the inspiratory gas mixture of neonates as well as the toxic effects of some metal ions are examples in which the role of oxidative stress as pathogenesis is well established [2]. For the general inflammatory reaction in critical illness resulting in multiple organ failure, oxidative stress is suggested to play a pivotal role, but the evidence for this hypothesis is not yet conclusive.

Several micronutrients have antioxidant properties; and, not uncommonly, deficiency symptoms in the case of a shortage of micronutrients are connected to oxidative stress [3]. Although several nutrients other than micronutrients also have antioxidant properties, a great deal of interest has focused on the role of antioxidant micronutrients. A number of trace elements and some vitamins are of particular interest: selenium, manganese, copper, zinc, and vitamins A, C, and E. Most often,

*Correspondence: jan.wernerman@karolinska.se

Department of Anesthesia and Intensive Care Medicine, K32, Karolinska University Hospital Huddinge, 14186 Stockholm, Sweden several micronutrients are combined in what are commonly called antioxidant cocktails. These are popular as food additives among healthy individuals as prevention against disease and also in critically ill patients.

Efforts to use antioxidant micronutrients to treat sickness have, in general, not been particularly successful [4]. In critical illness, there are reports of statistical connections between low plasma levels of antioxidant micronutrients and unfavorable outcomes [5]. Therefore, it is not farfetched to hypothesize that supplementation with antioxidant micronutrients may be beneficial for critically ill patients. Consequently, two recent metaanalyses of available randomized controlled trials of antioxidant micronutrient supplementation are of particular interest $[6,7]$.

Both meta-analyses aim to focus on only antioxidant micronutrients, disregarding trials in which micronutrients are combined with nutrients that have antioxidant properties and that are not micronutrients. This complexity of the antioxidant defense system is definitely a complicating factor because the possible effect of a single antioxidant in isolation may differ from that in combination with other antioxidants. The effects may be additive or even synergistic. Another complicating factor is whether a low plasma concentration really is indicative of depletion and an insufficient antioxidant defense in critical illness. Although it is well known that a general inflammation is associated with a lowering of micronutrient plasma concentrations, the need for supplementation is not self-evident.

Because the majority of randomized controlled trials included in the meta-analyses use a combination of several antioxidant micronutrients, conclusions are difficult to draw. The meta-analysis tool is useful as it helps to give a systematic overview, but the heterogeneity of antioxidant cocktails, protocols, and patient case mix also illustrates the difficulty of making clinical recommendations. The three studies with the greatest impact in the Manzanares meta-analysis [6] illustrate this problem: one is a trial of selenium only [8], one is a trial of selenium and glutamine [9], and the third is a trial of vitamins $\mathrm{C}$ and $E[10]$. 
Both meta-analyses conclude that antioxidant micronutrients are of potential benefit for critically ill patients and that larger prospective clinical trials are needed. It is easy to agree with the conclusion that supplementation with antioxidant micronutrients may be advantageous for critically ill patients. However, for any recommendation to change practice, it is necessary to scrutinize the safety of such supplementations. As the signal of an advantageous effect comes from a heterogeneous group of studies, conclusions regarding safety are particularly difficult to draw. This is further complicated by the different cocktails that are used. The recommendation to start up more large prospective trials must be well thought out before they are launched. The present state of knowledge is compromised by the heterogeneity of cocktails and protocols of existing studies. The scientific step to take is to gain more knowledge of mechanisms before running ahead with new randomized controlled trials using new cocktails. Instead, the use of well-defined pharmacological principles coupling plasma concentrations (or even better concentrations at active sites if possible) to effects and then to outcomes should be recommended.

In summary, while waiting for a more scientific approach to the use of antioxidant micronutrients, the meta-analyses presented are very helpful in systemizing the evidence contained in randomized controlled clinical trials. Antioxidant micronutrient supplementation will be used in clinical practice, but safety should be the focus. New studies should focus on elucidating the effects of the different components of antioxidant cocktails and linking effects to concentrations.

\section{Competing interests}

The author declares that he has no competing interests.

Published: 10 May 2012

\section{References}

1. Sies H (ed.): Oxidative Stress. New York: Academic Press; 1985.

2. Preiser JC: Oxidative stress. JPEN J Parenter Enteral Nutr 2012, 36:147-154.

3. Shenkin A: Micronutrients in health and disease. Postgrad Med J 2006, 82:559-567.

4. Halliwell B: The antioxidant paradox. Lancet 2000, 355:1179-1180.

5. Manzanares W, Biestro A, Galusso F, Torre MH, Mañay N, Pittini G, Facchin G, Hardy G: Serum selenium and glutathione peroxidase-3 activity: biomarkers of systemic inflammation in the critically ill? Intensive Care Med 2009, 35:882-889.

6. Manzanares W, Dhaliwal R, Jiang X, Murch L, Heyland DK: Antioxidant micronutrients in the critically ill: a systematic review and meta-analysis. Crit Care 2012, 16:R66.

7. Visser J, Labadarios D, Blaauw R: Micronutrient supplementation for critically ill adults: a systematic review and meta-analysis. Nutrition 2011 27:745-758.

8. Angstwurm MW, Engelmann L, Zimmermann T, Lehmann C, Spes CH, Abel P, Strauss R, Meier-Hellmann A, Insel R, Radke J, Schüttler J, Gärtner R: Selenium in Intensive Care (SIC): results of a prospective randomized, placebocontrolled, multiple-center study in patients with severe systemic inflammatory response syndrome, sepsis, and septic shock. Crit Care Med 2007, 35:118-126.

9. Andrews PJ, Avenell A, Noble DW, Campbell MK, Croal BL, Simpson WG, Vale LD, Battison CG, Jenkinson DJ, Cook JA; Scottish Intensive care Glutamine or seleNium Evaluative Trial Trials Group: Randomised trial of glutamine, selenium, or both, to supplement parenteral nutrition for critically ill patients. BMJ 2011, 342:d1542.

10. Crimi E, Liguori A, Condorelli M, Cioffi M, Astuto M, Bontempo P, Pignalosa O, Vietri MT, Molinari AM, Sica V, Della Corte F, Napoli C: The beneficial effects of antioxidant supplementation in enteral feeding in critically ill patients: a prospective, randomized, double-blind, placebo-controlled trial. Anesth Analg 2004, 99:857-863, table of contents.

doi:10.1186/cc11319

Cite this article as: Wernerman J: Micronutrients against oxidative stress time for clinical recommendations?. Critical Care 2012, 16:124. 